International Mathematical Forum, Vol. 9, 2014, no. 25, 1229 - 1236

HIKARI Ltd, www.m-hikari.com

http://dx.doi.org/10.12988/imf.2014.46121

\title{
On the Curves of Constant Breadth in Four Dimensional Galilean Space
}

\author{
Abdullah Mağden \\ Atatürk University, Faculty of Art and Sciences \\ Erzurum, Turkey \\ Süha Yılmaz \\ Dokuz Eylül University, Buca Educational Faculty \\ Buca-Izmir, Turkey
}

Copyright (C) 2014 Abdullah Mağden and Süha Yılmaz. This is an open access article distributed under the Creative Commons Attribution License, which permits unrestricted use, distribution, and reproduction in any medium, provided the original work is properly cited.

\begin{abstract}
In this work, we obtained characterization curves of constant breadth in four dimensional Galilean space in terms of Frenet-Serret vector fields. First we investigate an explicit characterization of curves of constant breadth of four dimensional Galilean space.In addition,it has been observed that differential equation of 4 th order of a constant breadth curve in four dimensional Galilean space.Finally,some differential and integral characterizations of the mentioned curves are expressed by the classical differential geometry methods.
\end{abstract}

\section{Introduction}

Curves of constant breadth were introduced by L. Euler in 1870. Ö. Köse (1984) wrote some geometric properties of plane curves of constant breadth. And, in another work Ö. Köse (1986) extended these properties to the Euclidian 3-space $\mathrm{E}^{3}$. F. Reuleaux gave a method obtaining some curves of costant breadth and found use in the kinematies of machinary. 
Furthermore, W. Blaschke defined the curve of costant breadth on the sphere and M. Fujivara had obtained a problem to determine whether there exist "space curve of constant breadth" or not, and he defined "breadth" for space curves and obtained these curves on a surface of constant breadth. A. Mağden and Ö. Köse (1997) studied this kind curves in four dimensional Euclidean space $E^{4}$. S. Yllmaz and M. Turgut expressed some characterizations of timelike curves of constant breadth in Minkowski 3-space and partially null curves of constant breadth in semi-Riemannian space in [14-15]. And recently this topic were studied in higher dimensions and further characterizations related to different geometries were obtained, see [1], [4], [6-8] and [11-13],S.Yılmaz (2010) defined Frenet-Serret frame in four dimensional Galilean Space,see[16].

Galilean geometry makes its extensive development an easy matter,and extensive development of a new geometric system is aprecondition for an effective comparison of it with Euclidean geometry.Also,extensive development is likely to give the student the psychological assurance of the consistency of the investigated structure. Another distinction of Galilean geometry is the fact that it exemplifies the fruitful geometric idea of duality,see [17]. The populer name "Galilean geometry" is historically in acceurate: Galilean, whose works date from the beginning of the 17 th century, did not in fact know this geometry, whose discovery was necessarily preceded by one of the greatest intellectual triumphs of the 19 th century - the emergence of the idea that many legitimate geometric systems exist. A more accurate name would be "the geometry associated with the Galilean principle of the relativity".

\section{Preliminaries}

The study of mechanics of plane-parallel motions reduces to the study of a geometry of three dimensional space with coordinates $\{x, y, t\}$ is given by the motion formula Yaglom(1979).

$$
\begin{aligned}
x^{\prime} & =(\cos \alpha) x+(\sin \alpha) y+(v \cos \beta) t+a, \\
y^{\prime} & =-(\sin \alpha) x+(\cos \alpha) y+(v \sin \beta) t+b \\
t^{\prime} & =t+d .
\end{aligned}
$$

This geometry can be called three-dimensional Galilean Geometry. Yaglom (1979) stressed that four-dimensional Galilean Geometry, which studies all properties invariant under motions of objects in space, is even more complex. Yaglom(1979) also stated this geometry can be described more precisely as the study of those properties of four-dimensional space with coordinates that are invariant under the general Galilean transformations.

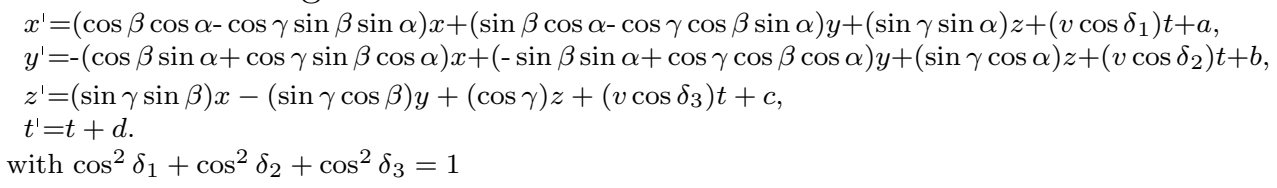


In the light of the motion equations above, we observe that the geometry of four-dimensional Galilean geometry roughly restricted.In this work, we intend to study theory of constant breadth of curves expressed in Röschel(1986) and Öğrenmiş et al.(2009) to the Galilean 4-space.

Let $\alpha: I \subset R \longrightarrow G^{4}$ be a curve given by

$\alpha(t)=(x(t), y(t), z(t), w(t))$,

where $x(t), y(t), z(t), w(t) \in C^{4}$ (the set of three times continuously differentiable functions) and t run through in a real interval.Let $\alpha$ be a curve in $G^{4}$,parameterized by arc length $\mathrm{t}=$ s, given coordinate form

$\alpha(s)=(s, y(s), z(s), w(s))$

In affine coordinates between two points the scalar product is defined by

$$
g\left(P_{1}, P_{2}\right)=\left\{\begin{array}{ll}
\left|x_{21}-x_{11}\right| & , \text { if } x_{11} \neq x_{21} \\
\sqrt{\left(x_{22}-x_{12}\right)^{2}+\left(x_{23}-x_{13}\right)^{2}+\left(x_{24}-x_{14}\right)^{2}} & , \text { if } x_{11}=x_{21}
\end{array}\right\} .
$$

where, $P_{i}=\left(x_{i 1}, x_{i 2}, x_{i 3}, x_{i 4}\right), i=1,2$

We define the Galilean cross product in $G^{4}$ for the vectors $x=\left(x_{1}, x_{2}\right.$, $\left.x_{3}, x_{4}\right)$ and $y=\left(y_{1}, y_{2}, y_{3}, y_{4}\right)$ and $z=\left(z_{1}, z_{2}, z_{3}, z_{4}\right)$ as follows:

$$
x \wedge y \Lambda z=\left|\begin{array}{cccc}
0 & e_{2} & e_{3} & e_{4} \\
x_{1} & x_{2} & x_{3} & x_{4} \\
y_{1} & y_{2} & y_{3} & y_{4} \\
z_{1} & z_{2} & z_{3} & z_{4}
\end{array}\right|
$$

where $e_{i}$ are standard basis vectors. $(\mathrm{i}=1,2,3,4)$

Let $\alpha(s)=(s, y(s), z(s), w(s))$ be a curve parametrized by arc length $\mathrm{s}$ in $G^{4}$.Here,we denote differentiation with respect to s by a dash. The real valued function

$\kappa(\mathrm{s})=\left\|t^{\prime}\right\|=\sqrt{\left(y^{\prime \prime}(s)\right)^{2}+\left(z^{\prime \prime}(s)\right)^{2}+(w(s))^{2}}$

is called the first curvature of the curve $\alpha$.we define the second and third curvature function as, respectively

$\tau(s)=\left\|n^{\prime}(s)\right\|$

$\sigma(s)=\left\langle b^{\prime}, e\right\rangle_{G}$

In the rest of the paper, we shall suppose $\kappa(\mathrm{s}) \neq 0, \tau(\mathrm{s}) \neq 0, \sigma(\mathrm{s}) \neq 0$ at everywhere.

The orthonormal quad of the curve $\alpha$ is defined by

$$
\begin{aligned}
& t(s)=\alpha^{\prime}(s)=\left(1, y^{\prime}(s), z^{\prime}(s), w^{\prime}(s)\right) \\
& n(s) \frac{1}{\kappa(s)}\left(0, y^{\prime \prime}(s), z^{\prime \prime}(s), w^{\prime \prime}(s)\right) \\
& \left.\left.\left.b(s)=\frac{1}{\tau(s)}\left(0,\left(\frac{1}{\kappa(s)} y^{\prime \prime}(s)\right)\right)^{\prime},\left(\frac{1}{\kappa(s)} z^{\prime \prime}(s)\right)\right)^{\prime},\left(\frac{1}{\kappa(s)} w^{\prime \prime}(s)\right)\right)^{\prime} \cdot\right) \\
& e(s)=\mu t \wedge n \Lambda b .
\end{aligned}
$$

Here the coefficient $\mu$ is taken \pm 1 to make +1 the matrix $[t, n, b, e]$ 
The vectors $t(s), n(s), b(s)$ are called the tangent, principal normal ,the binormal line and trinormal line respectively. For their derivatives, the following Frenet formulas hold

$$
\begin{aligned}
& t^{\prime}(s)=\kappa(s) n(s) \\
& n^{\prime}(s)=\tau(s) b(s) \\
& b^{\prime}(s)=-\tau(s) n(s)+\sigma(s) e(s) \\
& e^{\prime}(s)=\sigma(s) b(s) \\
& \text { see[16]. }
\end{aligned}
$$

\section{Curves of Constant Breadth in Galilean Space $G^{4}$}

Let $\vec{\varphi}=\vec{\varphi}(s)$ and $\vec{\varphi}^{*}=\vec{\varphi}^{*}(s)$ be simple closed curve in Galilean space $G^{4}$. These curves will be denoted by $C$. The normal plane at every point $P$ on the curve meets the curve at a single point $\mathrm{Q}$ other then P.We call the point $\mathrm{Q}$ the opposite point of P.We consider a curve in the class $\Gamma$ as in $\mathrm{M}$. Fujivara (1914) having parallel tangents $\overrightarrow{\mathbf{T}}$ and $\overrightarrow{\mathbf{T}}^{*}$ in opposite directions at the opposite points $\varphi$ and $\varphi^{*}$ of the curve. A simple closed curve of constant breadth having parallel tangents in opposite directions at opposite points can be represented with respect to Frenet frame by the equation

$$
\vec{\varphi}^{*}=\vec{\varphi}+m_{1} \vec{T}+m_{2} \vec{N}+m_{3} \vec{B}+m_{4} \vec{E}
$$

where $m_{i}(s), 1 \leq i \leq 4$ arbitrary functions of $s$ and $\vec{\varphi}$ and $\vec{\varphi}^{*}$ are opposite points. Vector $d=\vec{\varphi}^{*}-\vec{\varphi}$ is called "the distance vector" of $C$. Differentiating both sides of (3.1) and considering Frenet equations, we have

$$
\begin{aligned}
\frac{d \varphi^{*}}{d s}=\vec{T}^{*} \frac{d s^{*}}{d s}= & \left(1+\frac{d m_{1}}{d s}\right) \vec{T}+\left(m_{1} \kappa+\frac{d m_{2}}{d s}-m_{3} \tau\right) \vec{N} \\
& +\left(m_{2} \tau+\frac{d m_{3}}{d s}-m_{4} \sigma\right) \vec{B}+\left(m_{3} \sigma+\frac{d m_{4}}{d s}\right) \vec{E}
\end{aligned}
$$


Since $\vec{T}^{*}=-\vec{T}$. Rewriting (3.2) we obtain following system of equations,

$$
\begin{aligned}
& 1+\frac{d m_{1}}{d s}+\frac{d s^{*}}{d s}=0 \\
& m_{1} \kappa+\frac{d m_{2}}{d s}-m_{3} \tau=0 \\
& m_{2} \tau+\frac{d m_{3}}{d s}-m_{4} \sigma=0 \\
& m_{3} \sigma+\frac{d m_{4}}{d s}=0
\end{aligned}
$$

If we call $\theta$ as the angle between the tangent of the curve $C$ at point $\vec{\varphi}$ with a given fixed direction and $s$ arc length parameter of $\vec{\varphi}(s)$, consider $\frac{d \theta}{d s}=\kappa$, we have (3.3) as following;

$$
\begin{array}{ll}
\frac{d m_{1}}{d \theta} & =f(\theta), \\
\frac{d m_{2}}{d \theta}= & -m_{1}+m_{3} \rho \tau \\
\frac{d m_{3}}{d \theta}= & -m_{2} \rho \tau+m_{4} \rho \sigma \\
\frac{d m_{4}}{d \theta} & -m_{3} \rho \sigma
\end{array}
$$

where $f(\theta)=\rho+\rho^{*}, \rho=\frac{1}{\kappa}$ and $\rho^{*}=\frac{1}{\kappa^{*}}$ denote the radius of curvature at $\vec{\varphi}$ and $\vec{\varphi}^{*}$, respectively. If $m_{1}, m_{3}$ and their derivates are eliminated in equations (3.4), we obtain the following equation with respect to $m_{2}$ :

$$
\begin{aligned}
& \frac{d^{4} m_{2}}{d \theta^{4}}-\left[\left[\ln (\rho \tau)^{\prime}\right] \cdot \frac{d f}{d \theta} \cdot H(\theta)\right] \frac{d^{3} m_{2}}{d \theta^{3}}+\left\{(\rho \tau)^{2^{\prime}}+(\rho \tau)^{2}+\rho^{2} \sigma^{2}+\left[2(\ln (\rho \tau)]^{\prime \prime}\right\} \frac{d^{2} m_{2}}{d \theta^{2}}\right. \\
& +\left[2(\rho \tau)^{2^{\prime}}+\left(\rho^{2} \sigma^{2}\right)^{\prime}+\left(\rho^{2} \tau \sigma\right)^{\prime}\right] \frac{d m_{2}}{d \theta}+\left[(\rho \tau)^{2^{\prime \prime}}+\left[\ln (\rho \tau)^{\prime \prime \prime}\right]\right] m_{2}+\frac{d^{2} f}{d \theta^{2}}-\left[\ln (\rho \tau)^{\prime}\right] \frac{d f}{d \theta} \\
& +\left\{\rho^{2} \sigma^{2}+\left[(\ln (\rho \tau)]^{\prime \prime}\right\} f+\left(\rho^{2} \sigma^{2}\right)^{\prime}+\frac{\sigma}{\tau}\left(\rho^{2} \tau \sigma\right)+\left[\ln (\rho \tau)^{\prime \prime \prime}\right] F(\theta)+H(\theta)=0\right.
\end{aligned}
$$

where $H(\theta)=\int\left[\frac{d m_{2}}{d \theta}+\int f d \theta\right] \frac{\sigma}{\tau} d \theta, F(\theta)=\int f d \theta$

These equations are characterizations for the curve $\vec{\varphi}^{*}$. If the distance between opposite points of $C$ and $C^{*}$ is constant, then, due to null frame vectors, we can write that

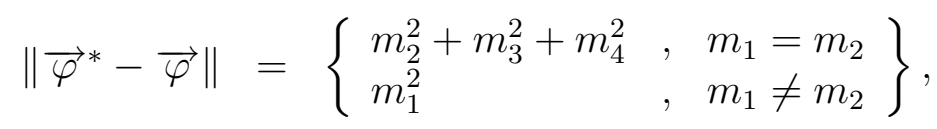


and if $m_{1}=m_{2}$, we can write that

$$
m_{2}^{2}+m_{3}^{2}+m_{4}^{2}=k^{2}=\text { constant } .
$$

Hence, by the differentiation we have

$$
m_{2} \frac{d m_{2}}{d \theta}+m_{3} \frac{d m_{3}}{d \theta}+m_{4} \frac{d m_{4}}{d \theta}=0 .
$$

Considering system (3.4), we obtain

$$
m_{1} \cdot m_{2}=0 .
$$

Therefore, we arrive at $m_{1}=0$ or $m_{2}=0$. As a next step, we shall study in the following cases.

Case 1: If $m_{1}=0$. then $f=0$, in this case, equation (3.5) gives

$$
\begin{aligned}
& \frac{d^{4} m_{2}}{d \theta^{4}}+\left\{(\rho \tau)^{2^{\prime}}+(\rho \tau)^{2}+\rho^{2} \sigma^{2}+\left[2(\ln (\rho \tau)]^{\prime \prime}\right\} \frac{d^{2} m_{2}}{d \theta^{2}}+\right. \\
& \quad+\left[2(\rho \tau)^{2^{\prime}}+\left(\rho^{2} \sigma^{2}\right)^{\prime}+\left(\rho^{2} \tau \sigma\right)^{\prime}\right] \frac{d m_{2}}{d \theta}+\left[(\rho \tau)^{2^{\prime \prime}}+\left[\ln (\rho \tau)^{\prime \prime \prime}\right]\right] m_{2}=0
\end{aligned}
$$

If $m_{1}$ is constant, besides, let us suppose that $m_{2}=0$. Then we obtain

$$
f(\theta)=0, \quad m_{3}=\frac{m_{1}}{\rho \tau}, m_{4}=-\int \rho \sigma m_{3} d \theta
$$

If $m_{1}=k_{1},\left(k_{1} \in \mathbb{R}\right)$, in this case equation (3.1) transforms to

$$
\vec{\varphi}^{*}=\vec{\varphi}+k_{1} \vec{T}+\frac{m_{1}}{\rho \tau} \vec{B} .-\left[\int \rho \sigma m_{3} d \theta\right] \vec{E}
$$

The change of variable $\mathrm{t}(\Psi)=\int_{0}^{\Psi} \rho \sigma d \Psi$ gives

$$
\frac{d^{2} m_{3}}{d t^{2}}+m_{3}=0
$$

General solution for $m_{3}(t)$ is

$$
m_{3}=A \cos \left(\int_{0}^{\Psi} \rho \sigma d t\right)+B \sin \left(\int_{0}^{\Psi} \rho \sigma d t\right)
$$

If we consider $m_{3}$, then

$$
m_{4}=-A \sin \left(\int_{0}^{\Psi} \rho \sigma d t\right)+B \cos \left(\int_{0}^{\Psi} \rho \sigma d t\right),
$$

where $\mathrm{A}$ and $\mathrm{B}$ are arbitrary constants. Therefore,the general solution set of the system

$$
\left\{\begin{array}{c}
m_{1}=k_{1},\left(k_{1} \in \mathbb{R}\right), \\
m_{2}=0, \\
m_{3}=A \cos \left(\int_{0}^{\Psi} \rho \sigma d t\right)+B \sin \left(\int_{0}^{\Psi} \rho \sigma d t\right), \\
m_{4}=-A \sin \left(\int_{0}^{\Psi} \rho \sigma d t\right)+B \cos \left(\int_{0}^{\Psi} \rho \sigma d t\right) .
\end{array}\right\}
$$


Thus equation (3.1) becomes

$$
\vec{\varphi}^{*}=\vec{\varphi}+k_{1} \vec{T}+\left\{A \cos \left(\int_{0}^{\Psi} \rho \sigma d t\right)+B \sin \left(\int_{0}^{\Psi} \rho \sigma d t\right)\right\} \vec{B}+\left\{-A \sin \left(\int_{0}^{\Psi} \rho \sigma d t\right)+B \cos \left(\int_{0}^{\Psi} \rho \sigma d t\right) \cdot\right\} \vec{E}
$$

Case 2: i) If $m_{2}=0$, then the equation (3.5) yields

$\frac{d^{2} f}{d \theta^{2}}-\left[\ln (\rho \tau)^{\prime}\right] \frac{d f}{d \theta}+\left\{\rho^{2} \sigma^{2}+\left[(\ln (\rho \tau)]^{\prime \prime}\right\} f+\left(\rho^{2} \sigma^{2}\right)^{\prime}+\frac{\sigma}{\tau}\left(\rho^{2} \tau \sigma\right)+\left[\ln (\rho \tau)^{\prime \prime \prime}\right] F(\theta)+H(\theta)=0\right.$

ii) if $m_{1} \neq m_{2}$, we get

$$
m_{1}^{2}=k_{1}^{2},
$$

where $k_{1}^{2} \in \mathbb{R}$. Hence, by the differentiation we have

$$
m_{1} \frac{d m_{1}}{d \theta}=0
$$

If we carry (3.4) into (3.14) we obtain same cases and equation (3.10).

\section{REFERENCES}

[1] Z. Akdogan, A. Măgden. Some characterizations of curves of constant breadth in $\mathrm{E}^{n}$ space. Turk. J. Math., 2001, 25: 433-444.

[2] W. Blaschke. Konvexe Bereiche gegebener konstanter Breite und kleinsten Inhalts. Math. Ann., 1915, 76: 504-513.

[3] B. Divjak. Curves in pseudo-Galilean Geometry. Annales Univ. Budapest, 1998, 41: 117-128.

[4] M. Ergüt, A.O. Öğrenmiş. Some characterizations of spherical curves in Galilean space. Journal of Advanced Research in Pure Mathematics, 2009, 1: $18-26$.

[5] M. Fujivara. On space curves of constant breadth. Tohoku Math. J., 1914, 5: 179-184.

[6] M. Kazaz, M. Onder, H. Kocayigit. Spacelike curves of constant breadth in Minkowski 4-space. Int. J. Math. Anal., 2008, 2: 1061-1068.

[7] H. Kocayigit, M. Onder. Space curves of constant breadth in Minkowski 3-space. Annali di Matematica, doi: 10.1007/s10231-011-0247-5.

[8] M. Onder, H. Kocayigit, E. Candan. Differential equations characterizing timelike and spacelike curves of constant breadth in Minkowski 3-space $\mathrm{E}_{1}^{3}$. J. Korean Math. Soc., 48: 849-866, doi: 10.4134/JKMS.2011.48.4.849.

[9] Ö. Köse. Some properties of ovals and curves of constant width in a plane. Doğa Math., 1984, 8: 119-126.

[10] Ö. Köse. On space curves of constant breadth. Doğa Math., 1986, 10 : $11-14$. 
[11] A. Mağden, Ö. Köse. On the curves of constant breadth in $\mathrm{E}^{4}$ space. Turk. J. Math., 1997, 21: 227-284.

[12] M. Sezer. Differential equations characterizing space curves of constant breadth and a criterion for these curves. Doğa Mat., 1989, 13: 70-78.

[13] M. Turgut. Smarandache breadth pseudo null curves in Minkowski space-time. International J. Math. Combin., 2009, 1: 46-49.

[14] S. Yllmaz, M. Turgut. On the time-like curves of constant breadth in Minkowski 3-Space. International J. Math. Combin., 2008, 3: 34-39.

[15] S. Yilmaz, M. Turgut. Partially null curves of constant breadth in semi-Riemannian space. Modern Applied Science, 2009, 3: 60-63.

[16] S. Yilmaz, Construction of the Frenet-Serret Frame of a curve in 4D Galilean space and some applications.International Journal of the Physical Sciences.,2010,5:1284-1289.

[17] I.M.Yaglom,A Simple Non-Euclidean Geometry and Its Physical Basis. Springer-Verlag,New York., 1979, Preface ix.

\section{Received: June 7, 2014}

